\begin{tabular}{|c|c|}
\hline Title & Computation of Macroscopic Electromagnetic Properties of Soft Magnetic Composite \\
\hline Author(s) & Ito, Y asuhisa; Igarashi, Hajime \\
\hline Citation & $\begin{array}{l}\text { IEEE T ransactions on Magnetics, 49(5), 1953-1956 } \\
\text { https://doi.org/10.1109// MA G.2013.2240444 }\end{array}$ \\
\hline Issue Date & $2013-05$ \\
\hline Doc URL & http://hdl.handle.net/2115/53412 \\
\hline Rights & $\begin{array}{l}\text { ( } 2013 \text { IEEE. Reprinted, with permission, from Y asuhisa Ito,Hajime Igarashi, Computation of Macroscopic } \\
\text { Electromagnetic Properties of Soft Magnetic Composite, IEEE Transactions on Magnetics, May. 2013. This material is } \\
\text { posted here with permission of the IEEE. Such permission of the IEEE does not in any way imply IEEE endorsement of } \\
\text { any of Hokkaido University products or services. Internal or personal use of this material is permitted. However, } \\
\text { permission to reprint/republish this material for advertising or promotional purposes or for creating new collective } \\
\text { works for resale or redistribution must be obtained from the IEEE by writing to pubs permissions@ ieee.org. By } \\
\text { choosing to view this document, you agree to all provisions of the copyright laws protecting it. }\end{array}$ \\
\hline Type & article (author version) \\
\hline File Information & Computation of Macroscopic Electromagnetic Properties of Soft Magnetic Composite.pdf \\
\hline
\end{tabular}

Instructions for use 


\title{
Computation of Macroscopic Electromagnetic Properties of Soft Magnetic Composite
}

\author{
Yasuhisa Ito ${ }^{1}$, Hajime Igarashi ${ }^{1}$, Member, IEEE \\ ${ }^{1}$ Graduate School of Information Science and Technology, Hokkaido University, \\ Kita 14, Nishi 9, Kita-ku, 060-0814, Sapporo, Japan
}

\begin{abstract}
This paper presents a new numerical method to compute the macroscopic electromagnetic properties of the soft magnetic composite (SMC). In present method, SMC is assumed to be composed of homogeneous magnetic bricks with periodicity. The macroscopic electromagnetic properties of SMC are obtained using finite element method in which it is assumed that the homogenized energy is equal to that stored in a unit domain. We compare the macroscopic electromagnetic properties obtained by the present method with those obtained by Ollendorf's formula and magnetic circuit based on the nonmagnetic grain boundary model. It is shown that the three results are in good agreement when assuming constant permeability. It is also found that there are clear discrepancies among them due to magnetic saturations especially when the demagnetization coefficient is large.
\end{abstract}

Index Terms-Soft magnetic composite, Finite element method, Ollendorf's formula, Nonmagnetic grain boundary model, Inductor

\section{INTRODUCTION}

$\mathrm{S}_{\mathrm{n}}^{\mathrm{s}}$ OFT MAGNETIC COMPOSITE (SMC) CORES composed of $\gamma_{\text {magnetic particles coated by electrically insulating and }}$ non-magnetic material have advantages over traditional laminated steels used in electromagnetic machines such as motors and reactors [1], [2]: the former has lower eddy current losses and is adequate for forming 3D shapes [3]. It is experimentally shown that the macroscopic permeability and eddy current losses in SMC depend on the density, size and shape of the magnetic particles. Therefore it is necessary to optimize these parameters to realize desirable properties for SMC such as high permeability and low eddy current losses. The macroscopic electromagnetic properties of SMC can approximately be estimated by the classical methods based on Maxwell-Gamett's [4], [5], Ollendorf's [6] and Bruggeman's formulae [7]. The permeability of SMC can also be modeled using a magnetic circuit method based on the nonmagnetic grain boundary (NMGB) model [8]. However these formulae would not be valid for saturated cores, and these cannot treat with magnetic hysteresis and eddy current losses.

This paper presents a new numerical method based on finite element method (FEM) to compute the macroscopic electromagnetic properties of SMC. In the present method, it is assumed that SMC is composed of homogeneous magnetic bricks with periodicity. The macroscopic electromagnetic

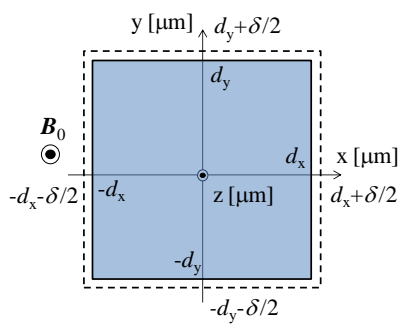

(a) $\mathrm{x}$-y plane

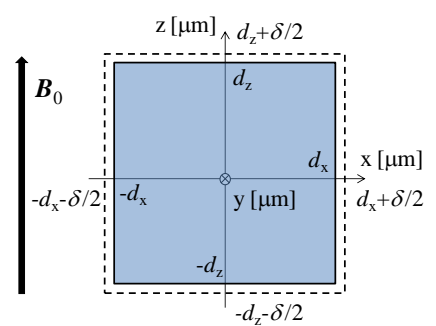

(b) X-z plane
Fig. 1 Model 1: unit domain of simple cubic lattice

Manuscript received November 10, 2012.

Corresponding author: Y. Ito (e-mail: yasuito@em-si.eng.hokudai.ac.jp).

Digital Object Identifier inserted by IEEE properties obtained by the present method in linear and nonlinear analysis are compared with these obtained by Ollendorf's formula and magnetic circuit model. Moreover we study the effect of the deviations in the volume fraction of magnetic particles in SMC on the magnetic property of the inductor on the basis of the present method.

\section{NUMERICAL METHOD}

\section{A. Analysis model}

Let us consider SMC which consists of magnetic particles coated by non-magnetic material with periodic configuration. We assume the brick-shaped particles of the same size for simplicity. The uniform magnetic induction $\boldsymbol{B}_{0}$ is applied to SMC in $z$-direction as shown in Fig. 1. We assume the Dirichlet and Neumann boundary condition on the side and top-bottom boundaries of the domain, respectively.

\section{B. Finite element formulation}

Let us consider static magnetic fields without electric currents. The governing equation is

$$
\nabla \times\left(\frac{1}{\mu} \nabla \times \boldsymbol{A}\right)=0
$$

where $\mu, \boldsymbol{A}$ are the permeability and vector potential, the later of which is discretized using the vector interpolation functions $N$ defined for each element edge as follows:

$$
\boldsymbol{A} \approx \sum_{\mathrm{j}} a_{\mathrm{j}} \boldsymbol{N}_{\mathrm{j}}
$$

where $\mathrm{j}$ denotes the edge index. The FE equation for (1) can be written in the form

$$
\mathrm{K}(\boldsymbol{a}) \boldsymbol{a}=0
$$

where

$$
K_{\mathrm{ij}}(\boldsymbol{a})=\int_{V}\left(\nabla \times \boldsymbol{N}_{\mathrm{i}}\right) \cdot\left(\frac{1}{\mu(\boldsymbol{a})} \nabla \times \boldsymbol{N}_{\mathrm{j}}\right) \mathrm{d} V .
$$

The unknown $\boldsymbol{a}$ is now divided into two parts: $\boldsymbol{a}=\boldsymbol{a}$ ' $+\boldsymbol{a}_{0}$ where $\boldsymbol{a}$ ' and $\boldsymbol{a}_{0}$ correspond to the induced and external vector potentials. When we assume constant permeability for the 
magnetic particles, (3) is rewritten as

$$
\mathrm{K}^{\prime}\left(\boldsymbol{a}^{\prime}\right) \boldsymbol{a}^{\prime}=-\mathrm{K} \boldsymbol{a}_{0}
$$

where $\mathrm{K}^{\prime}$ is the FE matrix to which the boundary conditions are applied.

When we consider the magnetic saturation in the magnetic particles, the Newton-Raphson method is employed to derive the linearized equation given by

$$
\boldsymbol{G}_{\mathrm{i}}\left(\boldsymbol{a}^{\prime}+\boldsymbol{a}_{0}+\Delta \boldsymbol{a}^{\prime}\right) \approx \boldsymbol{G}_{\mathrm{i}}(\boldsymbol{a})+\sum_{\mathrm{k}} \frac{\partial \boldsymbol{G}_{\mathrm{i}}(\boldsymbol{a})}{\partial a_{\mathrm{k}}^{\prime}} \Delta a_{\mathrm{k}}^{\prime}=0
$$

where

$$
\begin{gathered}
\boldsymbol{G}_{\mathrm{i}}(\boldsymbol{a})=\sum_{\mathrm{j}}\left(K_{\mathrm{ij}}^{\prime} \boldsymbol{a}_{\mathrm{j}}^{\prime}+K_{\mathrm{ij}} \boldsymbol{a}_{0 \mathrm{j}}\right), \\
\frac{\partial \boldsymbol{G}_{\mathrm{i}}(\boldsymbol{a})}{\partial a_{\mathrm{k}}^{\prime}}=\int_{V}\left(\nabla \times N_{\mathrm{i}}\right) \cdot\left(\frac{1}{\mu} \nabla \times \boldsymbol{N}_{\mathrm{k}}\right) \mathrm{d} V \\
+\sum_{\mathrm{j}} a_{\mathrm{j}} \int_{V} \frac{\partial v(\boldsymbol{a})}{\partial a_{\mathrm{k}}^{\prime}}\left(\nabla \times \boldsymbol{N}_{\mathrm{i}}\right) \cdot\left(\nabla \times \boldsymbol{N}_{\mathrm{j}}\right) \mathrm{d} V \\
+\sum_{\mathrm{j}} a_{0 \mathrm{j}} \int_{V} \frac{\partial v(\boldsymbol{a})}{\partial a_{\mathrm{k}}^{\prime}}\left(\nabla \times \boldsymbol{N}_{\mathrm{i}}\right) \cdot\left(\nabla \times \boldsymbol{N}_{\mathrm{j}}\right) \mathrm{d} V, \\
\frac{\partial v(\boldsymbol{a})}{\partial a_{\mathrm{k}}^{\prime}}=\frac{\partial v}{\partial B^{2}} \frac{\partial B^{2}}{\partial a_{\mathrm{k}}^{\prime}}=\frac{\partial v}{\partial B^{2}} \frac{\partial}{\partial a_{\mathrm{k}}^{\prime}}\left(\boldsymbol{B}^{\prime}+\boldsymbol{B}_{0}\right)^{2} \\
=2 \frac{\partial v}{\partial B^{2}}\left\{\sum_{\mathrm{m}} a_{\mathrm{m}}^{\prime}\left(\nabla \times \boldsymbol{N}_{\mathrm{k}}\right) \cdot\left(\nabla \times \boldsymbol{N}_{\mathrm{m}}\right)\right. \\
\left.+\sum_{\mathrm{n}} a_{0 \mathrm{n}}\left(\nabla \times \boldsymbol{N}_{\mathrm{k}}\right) \cdot\left(\nabla \times \boldsymbol{N}_{\mathrm{n}}\right)\right\} .
\end{gathered}
$$

The derivative of $v$ with respect to $B^{2}$ in (9) can be obtained from the $\mathrm{BH}$ curve.

\section{COMPUTATION OF MACROSCOPIC ElECTROMAGNETIC PROPERTIES}

\section{A. Linear analysis}

In the present method, the macroscopic permeability is obtained so that the magnetic energy in the unit domain, shown in Fig. 1, including the particle and non-magnetic layer is equal to the homogenized energy [9]. When assuming the constant permeability, the magnetic energy $W$ in a unit domain is expressed as

$$
W=\int_{V} \frac{1}{2} H B \mathrm{~d} V \approx \sum_{\mathrm{e}} \frac{1}{2} \frac{B_{\mathrm{e}}^{2}}{\mu_{\mathrm{e}}} \Delta v_{\mathrm{e}}
$$

where $V$, e, $\mu_{\mathrm{e}}$, and $\Delta v_{\mathrm{e}}$ are the volume of the unit domain, element index, permeability and volume of element e. The homogenized magnetic energy $W^{\prime}$ is expressed in terms of the average field $\bar{B}$ as follows:

$$
W^{\prime} \approx \frac{V}{2} \frac{\bar{B}^{2}}{\bar{\mu}} \approx \frac{V}{2 \bar{\mu}}\left(\frac{1}{V} \sum_{\mathrm{e}} B_{\mathrm{e}} \Delta v_{e}\right)^{2} .
$$

From (10) and (11), we can obtain the macroscopic permeability for the linear case as:

$$
\bar{\mu}=\frac{\left(\sum_{\mathrm{e}} B_{\mathrm{e}} \Delta v_{e}\right)^{2}}{V \sum_{\mathrm{e}} \frac{B_{\mathrm{e}}^{2}}{\mu_{\mathrm{e}}} \Delta v_{\mathrm{e}}} .
$$

\section{B. Nonlinear analysis}

When we consider magnetic saturation, the magnetic energy can be expressed as

$$
\begin{aligned}
W & \approx \sum_{\mathrm{e}} \int_{0}^{B_{0}} \frac{B_{\mathrm{e}}}{\mu\left(B_{\mathrm{e}}\right)} \mathrm{d} B_{\mathrm{e}} \Delta v_{\mathrm{e}} \\
& =\sum_{\mathrm{e}}\left(\int_{0}^{B^{1}} \frac{B_{\mathrm{e}}}{\mu\left(B_{\mathrm{e}}\right)} \mathrm{d} B_{\mathrm{e}}+\cdots+\int_{B^{\mathrm{s}-1}}^{B^{\mathrm{s}}=B_{0}} \frac{B_{\mathrm{e}}}{\mu\left(B_{\mathrm{e}}\right)} \mathrm{d} B_{\mathrm{e}}\right) \Delta v_{\mathrm{e}} \\
& =\sum_{\mathrm{e}} \sum_{\mathrm{t}=1}^{\mathrm{s}} \Delta W_{\mathrm{e}}^{\mathrm{t}}
\end{aligned}
$$

where

$$
\begin{aligned}
\Delta W_{\mathrm{e}}^{\mathrm{t}} & =\int_{B^{\mathrm{t}-1}}^{B^{\mathrm{t}}} \frac{B_{\mathrm{e}}}{\mu\left(B_{\mathrm{e}}\right)} \mathrm{d} B_{\mathrm{e}} \Delta v_{\mathrm{e}} \\
& \approx \frac{1}{2}\left(\frac{B_{\mathrm{e}}^{\mathrm{t}}}{\mu\left(B_{\mathrm{e}}^{\mathrm{t}}\right)}+\frac{B_{\mathrm{e}}^{\mathrm{t}-1}}{\mu\left(B_{\mathrm{e}}^{\mathrm{t}-1}\right)}\right)\left(B_{\mathrm{e}}^{\mathrm{t}}-B_{\mathrm{e}}^{\mathrm{t}-1}\right) \Delta v_{\mathrm{e}} .
\end{aligned}
$$

On the other hand, the homogenized magnetic energy can be expressed as follows:

$$
W^{\prime} \approx\left(\int_{0}^{B^{1}} \bar{H}(\bar{B}) \mathrm{d} \bar{B}+\cdots+\int_{B^{\mathrm{s}-1}}^{B^{\mathrm{s}}=B_{0}} \bar{H}(\bar{B}) \mathrm{d} \bar{B}\right) V=\sum_{\mathrm{t}=1}^{\mathrm{s}} \Delta W^{\prime \mathrm{t}}
$$

where

$$
\begin{gathered}
\Delta W^{\prime \mathrm{t}}=\int_{B^{\mathrm{t}-1}}^{B^{\mathrm{t}}} \bar{H}\left(\bar{B}^{\mathrm{t}}\right) \mathrm{d} \bar{B}^{\mathrm{t}} V \approx \int_{B^{\mathrm{t}-1}}^{B^{\mathrm{t}}} \bar{H}\left(\bar{B}^{\mathrm{t}}\right)\left(\bar{B}^{\mathrm{t}}-\bar{B}^{\mathrm{t}-1}\right) V, \\
\bar{B}^{\mathrm{t}}=\frac{1}{V} \sum_{\mathrm{e}} B_{\mathrm{e}}^{\mathrm{t}} \Delta v_{\mathrm{e}} .
\end{gathered}
$$

From (13) and (15), we can obtain the macroscopic constitutive relation for the non-linear case as

$$
\bar{H}\left(\bar{B}^{\mathrm{p}}\right)=\frac{2\left(\sum_{\mathrm{e}} \sum_{\mathrm{t}=1}^{\mathrm{p}} \Delta W_{\mathrm{e}}^{\mathrm{t}}-\sum_{\mathrm{t}=1}^{\mathrm{p}-1} \Delta W^{\prime \mathrm{t}}\right)}{V\left(\bar{B}^{\mathrm{p}}-\bar{B}^{\mathrm{p}-1}\right)}-\bar{H}\left(\bar{B}^{\mathrm{p}-1}\right)
$$

where $1 \leq \mathrm{p} \leq \mathrm{s}$.

\section{NUMERICAL RESULTS}

\section{A. Ollendorf's formula and magnetic circuit model}

To test the validity of the present method, we compare the linear macroscopic magnetic properties obtained by the present method with those obtained by Ollendorf's formula and the magnetic circuit model. Ollendorf's formula [6]

$$
\bar{\mu}=\mu_{0}+\eta \frac{\mu_{0}\left(\mu_{m}-\mu_{0}\right)}{N(1-\eta)\left(\mu_{m}-\mu_{0}\right)+\mu_{0}}
$$

has widely been used to estimate the permeability of heterogeneous structures, where $\mu_{0}, \mu_{\mathrm{m}}, \eta$ and $N$ are the permeability of vacuum and magnetic particles, volume fraction of magnetic particles and coefficient of diamagnetic 
field which satisfies $0 \leq N \leq 1$. In the method proposed in [8], the magnetic field in the unit domain is analyzed using the magnetic circuit method. In this method, for the unit domain shown in Fig. 1 composed of a square flake with edge $2 d_{\mathrm{z}}$ and thickness $2 d_{\mathrm{x}}, 2 d_{\mathrm{y}}$ surrounded by a gap of uniform thickness $\delta / 2$, the permeability is given by

$$
\bar{\mu}=\frac{2 d_{\mathrm{z}}+\delta}{\left(2 d_{\mathrm{x}}+\delta\right)\left(2 d_{\mathrm{y}}+\delta\right)} \frac{4 \mu_{0} \mu_{\mathrm{m}} d_{\mathrm{x}} d_{\mathrm{y}}}{2 \mu_{0} d_{\mathrm{z}}+\mu_{\mathrm{m}} \delta} .
$$

\section{B. Analysis of model 1: simple cubic model}

The magnetic property of SMC is analyzed by the three methods assuming that it is composed of the simple cubic lattice shown in Fig. 1, which is called model 1. In the analysis, we set $d_{\mathrm{x}}=d_{\mathrm{y}}=d_{\mathrm{z}}=50 \mu \mathrm{m}$ and $N=1 / 3$ is substituted to Ollendorf's formula because of the isotropic nature of the model. In the linear analysis, the relative permeability of magnetic particles is 1000. In order to obtain the relationship between the macroscopic permeability and volume fraction $\eta$, the gap length $\delta$ is changed. From the results shown in Fig. 2, we can see that the values of macroscopic permeability obtained by the three methods are in good agreement regardless of $\eta$.

Next we consider the nonlinear magnetic property of SMC, where the magnetic particles are assumed to obey the $\mathrm{BH}$ characteristics of ferrite. Fig. 3 shows the magnetic property of the homogeneous ferrite core as well as macroscopic magnetic properties of SMC obtained by Ollendorf's formula, the magnetic circuit model and present method when $\eta=0.90$. To compute the macroscopic permeability from the Ollendorf's formula and magnetic circuit model, the permeability of the ferrite, which is a function of $H$, is substituted into (19) and (20). On the other hand, the BH characteristic is fully considered in the field computation of the present method. As shown in Fig. 3(a), the three methods give almost identical macroscopic BH curves when $N=1 / 3$. However, it is found in Fig. 3(b) that when $N=0.05, d_{\mathrm{x}}=d_{\mathrm{y}}=10 \mu \mathrm{m}, d_{\mathrm{z}}=54 \mu \mathrm{m}$, the differences among them become significant. In particular, Ollendorf's formula yields higher values in $B$ in comparison with the other two methods. Moreover, the difference between the results obtained by the present method and magnetic circuit becomes large as $H$ increases. This would attribute to the fact that the magnetic field with magnetic saturations due to low $N$ and high $H$ cannot be well approximated by the magnetic circuit.

\section{Analysis of model 2: body-centered cubic model}

We next consider SMC composed of the body-centered cubic lattice shown in Fig. 4, which is called model 2 hereafter. The macroscopic permeability determined by the magnetic circuit model is given by [8], [10]

$$
\bar{\mu}=\frac{2 d_{\mathrm{z}}+\delta}{\left(2 d_{\mathrm{x}}+\delta\right)\left(2 d_{\mathrm{y}}+\delta\right)} \frac{1}{\left(R_{\mathrm{p}}+R_{\mathrm{gz}} \| R_{\mathrm{gx}}\right)}
$$

where $R_{\mathrm{p}}, R_{\mathrm{gz}}$ and $R_{\mathrm{gx}}$ are the reluctances of the particle and gap along the $z$ and $x$ axes which can be expressed by

$$
R_{\mathrm{p}}=\frac{d_{\mathrm{z}}}{2 \mu_{\mathrm{m}} d_{\mathrm{x}} d_{\mathrm{y}}}, R_{\mathrm{gz}}=\frac{\delta}{4 \mu_{0} d_{\mathrm{x}} d_{\mathrm{y}}},
$$

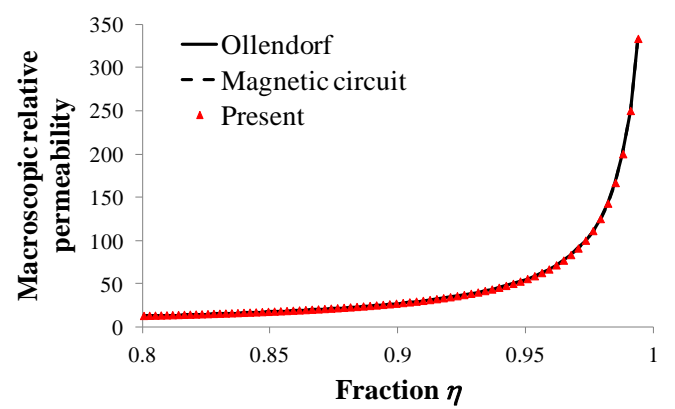

Fig. 2 Results in linear analysis of model 1

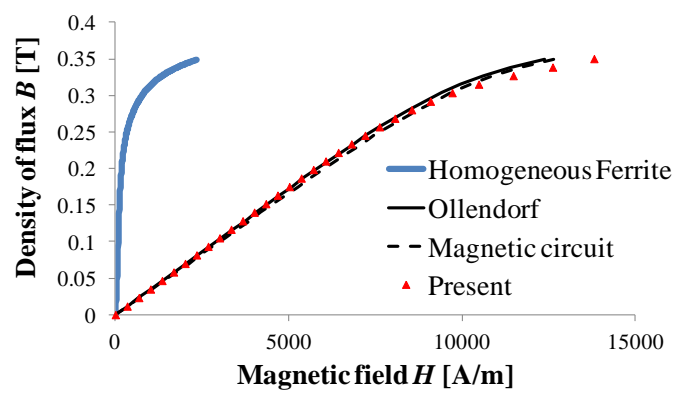

(a) $N=1 / 3$

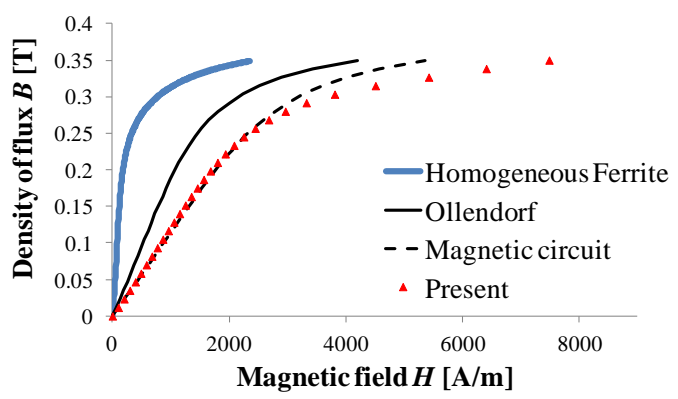

(b) $N=0.05$

Fig. 3 B-H curve in nonlinear analysis of model 1 when $\eta=0.90$

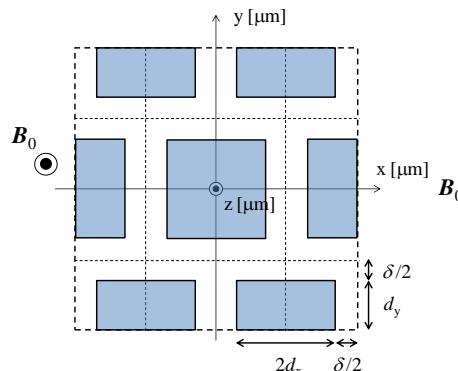

(a) $\mathrm{x}$-y plane

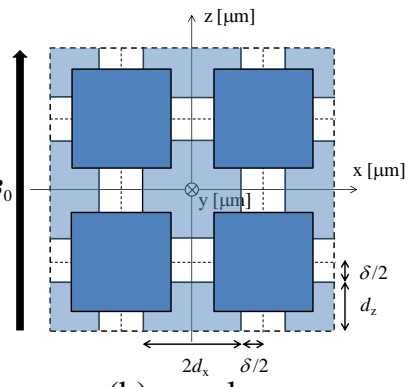

(b) X-z plane
Fig. 4 Model 2: unit domain of body-centered cubic lattice

$$
R_{\mathrm{gx}}=\frac{2 \delta}{\mu_{0}\left(2 d_{\mathrm{z}}-\delta\right)\left(2 d_{\mathrm{x}}-\delta\right)}+\frac{d_{\mathrm{z}}}{\mu_{\mathrm{m}}\left(2 d_{\mathrm{x}}-\delta\right) d_{\mathrm{y}}} .
$$

The length on the side of a magnetic particle in model 2 is set to $d_{\mathrm{x}}=d_{\mathrm{y}}=d_{\mathrm{z}}=50 \mu \mathrm{m}$ when $N=1 / 3, d_{\mathrm{x}}=d_{\mathrm{y}}=10 \mu \mathrm{m}, d_{\mathrm{z}}=54 \mu \mathrm{m}$ when $N=0.05$. The BH characteristics of ferrite and SMC modeled by the three methods are shown in Fig. 5. It can be seen in Fig. 5(a) that the computed macroscopic BH characteristics are somewhat similar to each other. However, as shown in Fig. 5(b), the discrepancies between the results obtained by the 


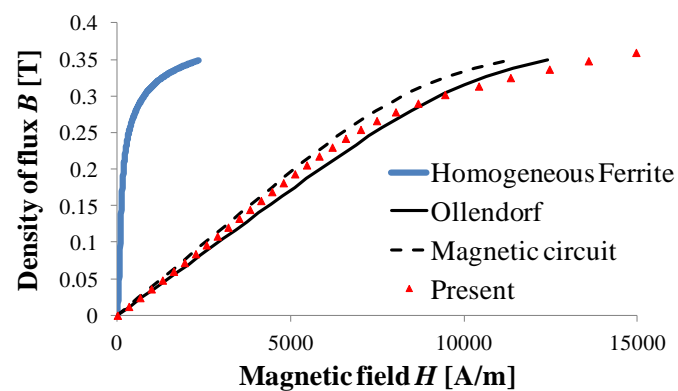

(a) $N=1 / 3$

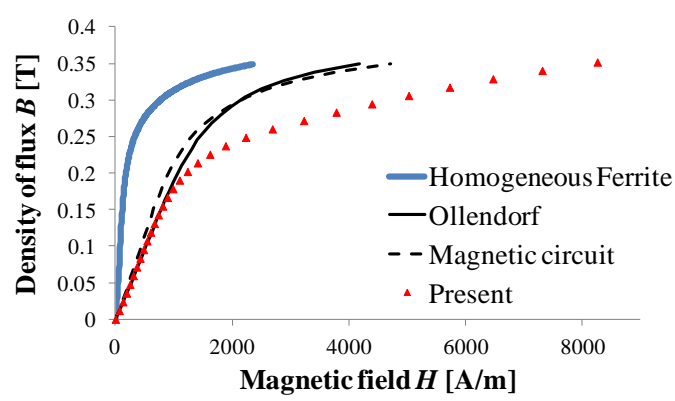

(b) $N=0.05$

Fig. 5 B-H curve in nonlinear analysis of model 2 when $\eta=0.90$

present method and the others become significant when $H$ increases. This would be due to the fact that the saturated magnetic fields cannot be accurately modeled by Ollendorf's approach and magnetic circuit method. Moreover, the differences in the results obtained by the three methods in model 2 are larger than those in model 1 . This would be due to the fact that magnetic resistivity in model 2 is lower than that in model 1 so that magnetic saturation easily occurs in the former.

\section{Analysis of inductor}

It is known that there is non-uniformity in the volume fraction $\eta$ of magnetic particles in SMC because of the stress in the production process. Here we analyze the effect of nonuniformity in $\eta$ on the magnetic property of the inductor using the present method in which model 1 is employed. The inductance of model inductor made of SMC shown in Fig. 6 is computed. In the analysis, the value of $\eta$ in a part of the upper magnetic plate is changed from 0.9 to 0.7 . The turn of coil is 1 and set to $1.0 \mathrm{~A}$. We find in Table 1 that the inductance becomes clearly small when there is non-uniformity in $\eta$.

\section{CONCLUSION}

In this paper, we have presented a new numerical method to compute the macroscopic electromagnetic properties of SMC. In the present method, these are obtained so that the magnetic energy in the unit domain including magnetic particles and non-magnetic layer is equal to the homogenized energy. The macroscopic magnetic properties obtained by the present method has been compared with these obtained by Ollendorf's formula and the magnetic circuit model. It has been found that the three methods give almost identical results for macroscopic permeability when the material is assumed to have linear $\mathrm{BH}$ property. On the other hand, there are significant differences among them especially when the demagnetization coefficient is large or the applied magnetic field is strong. The reason for the discrepancies would be due to the fact that the saturated magnetic fields cannot be well treated by Ollendorf's approach and magnetic circuit modeling. We plan to apply the present method to analysis of hysteresis and eddy current losses in SMC in future. Moreover, we will apply the present method to more complicated examples.

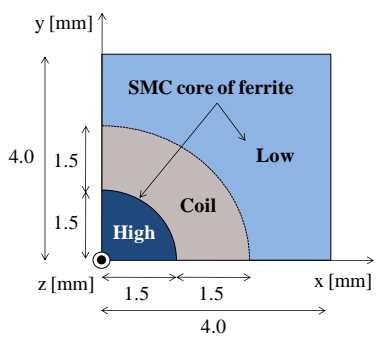

(a) $x-y$ plane

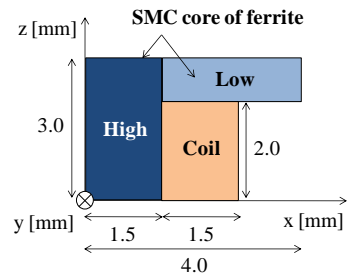

(b) X-z plane
Fig. 6 Model inductor of SMC

Table 1 Numerical results of the inductance

\begin{tabular}{ccc}
\hline \hline High fraction & Low fraction & Inductance $[\mu \mathrm{H}]$ \\
\hline Ferrite & Ferrite & 0.1750 \\
0.90 & 0.90 & 0.0798 \\
0.90 & 0.80 & 0.0698 \\
0.90 & 0.70 & 0.0623 \\
\hline \hline
\end{tabular}

\section{ACKNOWLEDGMENT}

Dr. K. Kawano of Taiyo Yuden Co. and Drs. A. Furuya, Y. Uehara of Fujitsu Ltd. gave us helpful comments for this study.

\section{REFERENCES}

[1] Y. G. Guo, J. G. Zhu, Z. W. Lin and J. J. Zhong, "Measurement and Modeling of Core Losses of Soft Magnetic Composites Under 3-D Magnetic Excitations in Rotating Motors," IEEE Trans. Magn., vol. 41, no. 10, pp. 3925-3927, 2005.

[2] M. Przybylski, B. Ślusarek, J. Gromek, "Brushless DC Motor with a Bonded Permanent Magnet and Powder Magnetic Core", XIX International Conference on Electrical Machines, pp. 1-4, 2010.

[3] H. Shokrollahi, K. Janghorban, "Soft magnetic composite materials (SMCs)," J. Mater. Process. Technol., 189, pp. 1-12, 2007.

[4] J. C. M. Garnett, "Colors in Material Glasses and Metal Films," Trans. Roy. Soc., vol. 53, pp. 385-420, 1904.

[5] A. Sihvola, "Electromagnetic Mixing Formulas and Applications," IEE ELECTROMAGNETIC WAVES SERIES 47, 1999.

[6] F. Ollendorf, "Magnetostatik der Massekerne," Arch. f. Eledtrotechnik., 25, pp. 436-447, 1931.

[7] D. A. G. Bruggeman, "Berechnung verschiedener physikalischer Konstanten von hetrogenen Substanzen, I. Dielektrizitatskonstanten und Leitfahigkeiten der Mischkorper aus isotropen Substanzen," Annalen der Physik, 5. Folge, Band 24, pp. 636-664, 1935.

[8] F. Mazaleyrat, V. Leger, R. Lebourgeois, and R. Barrue, "Permeability of Soft Magnetic Composites From Flakes of Nanocrystalline Ribbon," IEEE Trans. Magn., vol. 38, no. 5, pp. 3132-3134, 2002.

[9] H. Waki, H. Igarashi and T. Honma, "Estimation of Effective Permeability of Magnetic Composite Materials," IEEE Trans. Magn., vol. 41, no. 5, pp. 1520-1523, 2005.

[10] S. Handa, Y. Oshima and K. Akaiwa, "Effects of Particle Shapes on DCBiased Properties of Powder Cores and Modeling," J. Magn. Soc. Jpn., 35, pp. 436-442, 2011. 